\title{
MEDICAMENTOS PROVENIENTES DE FARMÁCIAS FITOTERAPÊUTICAS USADOS EM MUNICÍPIOS DA REGIÃO OESTE DO ESTADO DO RIO GRANDE DO NORTE
}

\author{
Antonia Mirian Nogueira de Moura Guerra. \\ Aluna de Graduação em Agronomia da UFERSA, Bolsista de Iniciação Científica - CNPq, Mossoró - RN \\ mirianagronoma@hotmail.com \\ Marcos de Freitas Pessoa. \\ Aluno de Graduação em Agronomia da UFERSA, Mossoró - RN. \\ marcos-fpessoa@hotmail.com
}

Julianna Vanessa de Assis Dantas Marques.

Aluna de Graduação em Agronomia da UFERSA, Mossoró - RN. julianna@alunos.esam.br

Celso Fabrício de Araújo.

Fitoterapêuta da Associação de Produtos Naturais Longa Vida. End.: Rua Pedro Abílio nº 738 - Umarizal/RN, telefone(84) 99758254, e-mail: sabemuito@linkmais.com.br.

Glênio Jadson Câncio Soares

Aluno de Graduação em Agronomia da UFERSA, Mossoró - RN

Glenio@hotmail.com

\begin{abstract}
RESUMO: O Brasil apresenta uma das maiores biodiversidades vegetal da Terra, e pelo menos a metade das espécies vegetais pode possuir alguma propriedade terapêutica útil à população, porém, uma pequena parte destas plantas foi estudada. Nas farmácias-vivas, os medicamentos são preparados em laboratório de fitoterápicos sob responsabilidade de um farmacêutico especialmente treinado. O objetivo deste estudo foi avaliar o uso destes medicamentos por parte das populações de municípios da região Oeste do Rio Grande do Norte, através da aplicação de questionários nos municípios de Campo Grande, Caraúbas, Martins e Umarizal. Em todos os municípios a população utiliza remédios fitoterápicos. A maioria dos clientes e usuários são mulheres numa faixa etária de 21-30 anos. 65\% dos entrevistados usaram e usam estes medicamentos. Nos municípios de Campo Grande, Caraúbas e Umarizal as classes sociais beneficiadas são média e baixa, enquanto que em Martins a classe beneficiada é a alta com 75\% dos clientes.
\end{abstract}

Palavras Chave: medicamento, farmácias fitoterápicas, uso.

\section{MEDICAMENTS PROVENEINT OF PHITOTHERAPIC PHARMACY USED IN MUNICIPALITY OF THE WEST REGION OF THE RIO GRANDE DO NORTE STATE.}

\begin{abstract}
The Brazil presents one of the biggest vegetal biodiversity on Earth, and at least half of the vegetable species have chances of having therapeutical properties useful to the population, but, few is part were studied. In the cheer-pharmacies, the medicaments are prepared in a phitotherapic laboratory under responsibility of a trained pharmaceutic. The objective of this study was to evaluate the use of this medicaments by the population of municipalities of the west region of the Rio Grande do Norte, affixing questionnaires on the municipalities of Campo Grande, Caraúbas, Martins and Umarizal. In all the municipalities, the population make use of phitotherapics medicaments. Most of the users and clients are women with age between 21 - 30 years. $65 \%$ of the interviewed people used and use these medicaments. In the municipalities of Campo Grande, Caraúbas and Umarizal, the benefited social classes are medium and the low, while in Martins, the benefited is the high, with $75 \%$ of the clients.
\end{abstract}

Key words: medicaments, phitotherapic pharmacy, use.

INTRODUÇÃo
As plantas medicinais desde os primórdios já eram utilizadas para o tratamento de moléstias do homem (HARRI et al. 2002). 


\section{REVISTA VERDE DE AGROECOLOGIA E DESENVOLVIMENTO SUSTENTÁVEL GRUPO VERDE DE AGRICULTURA ALTERNATIVA (GVAA)}

A utilização de plantas medicinais é uma prática generalizada na medicina popular. É o resultado do acúmulo de conhecimentos empíricos sobre a ação dos vegetais, por diversos grupos étnicos. Observações realizadas até agora permitem supor que todas as formações culturais fazem uso de plantas como recurso medicinal. No Brasil, além da assimilação dos conhecimentos indígenas, as contribuições trazidas pelos escravos e imigrantes representam papel importante para o surgimento de uma medicina popular rica e original, na qual a utilização de plantas medicinais ocupa lugar de destaque. Hoje, o seu uso não se restringe às zonas rurais ou regiões desprovidas de assistência médica e farmacêutica. Tudo indica que elas são utilizadas intensamente no meio urbano, como forma alternativa ou complementar aos medicamentos da medicina oficial (SIMÕES et al., 1989).

Conhecimentos, que a cargo dos anciãos eram transmitidos às novas gerações. Hoje não é diferente, os idosos são detentores de um vasto conhecimento adquirido ao longo da vida e os transmitem aos filhos e netos. Grandes conhecedores e consumidores de plantas medicinais, o idoso às utiliza para uso interno e externo difundindo entre os demais membros da família, principalmente, em comunidades rurais como produto terapêutico (MARTINS et al., 1995).

Entretanto, ultimamente este hábito não está sendo praticado apenas por idosos, é crescente o número de pessoas jovens e adultas, na faixa etária entre 20 e 45 anos que a cada dia fazem uso freqüente de plantas medicinais para cura de doenças mais comuns. Com os avanços ocorridos quanto ao desenvolvimento e divulgação de medicamentos fitoterápicos, a demanda por estes tem se tornado mais comum e freqüente entre as pessoas.

O comércio e o uso de plantas medicinais são bastante conhecidos e discutidos no Brasil e no mundo. $\mathrm{O}$ mercado atende de diferentes formas o consumidor desse tipo de produto, incluindo as comercializações feitas em empresas, em mercados e em ervanários (BRANDÃO; FREIRE; VIANNA-SOARES, 1998).

Nas farmácias-vivas, os medicamentos são preparados em laboratório de fitoterápicos sob responsabilidade de um farmacêutico especialmente treinado. Para sua administração, o princípio ativo é mantido nas plantas (e não isolado como faz a indústria farmacêutica) na forma de chás, xaropes, tinturas e cápsulas gelatinosas.

Estudos quanto à prática popular do uso de plantas medicinais para cura de doenças têm se tornado mais freqüentes e promissores. Conforme Carvalho et al., 2005, em estudo avaliando quais as espécies que eram utilizadas pela população de cidades do norte do estado do Paraná, concluiu que as espécies mais utilizadas são: hortelã, boldo, erva cidreira, erva doce e poejo, o que mostra que mesmo entre regiões diferentes pode haver uma semelhança quanto às preferências e uso das espécies medicinais, além disso, constatou um crescente interesse das pessoas entrevistadas em fazer em suas residências farmácias-vivas para terem disponíveis a todo instante algumas destas plantas para uso.

A utilização de fitoterápicos tem tido uma relevância sócio-econômica muito grande na qualidade de vida das comunidades de baixa renda dada a sua alta disponibilidade, baixa toxicidade, risco mínimo de efeitos colaterais e baixos custos e/ou sem ônus comparados aos medicamentos alopáticos (HOAREAU e DASILVA, 1999; RODRIGUES e CARVALHO, 2001).

Tendo em vista a importância das plantas medicinais e dos possíveis remédios desenvolvidos a partir destas, objetivou-se fazer um estudo de conhecimento acerca do uso destes medicamentos por parte da população dos municípios de Campo Grande, Caraúbas, Umarizal e Martins onde estão localizadas as farmácias fitoterápicas.

\section{MATERIAL E MÉTODOS}

A coleta de dados foi realizada através da aplicação de questionários semi-estruturados a 160 clientes, sendo 40 questionários em cada farmácia fitoterápica de cada município durante os meses de janeiro e fevereiro de 2006.

Foram escolhidos os municípios de Campo Grande, Caraúbas, Martins e Umarizal que estão localizados na região do Médio e Alto Oeste do Rio Grande do Norte, a escolha deu-se pelo fato destes serem os principais municípios da região que têm na prática da medicina popular através da presença de farmácias fitoterápicas um reforço ao uso de medicamentos naturais para cura das suas enfermidades mais comuns. O questionário semi-estruturado utilizado para coleta de informações continha dados acerca do sexo do cliente, idade, usuário, freqüência de uso e classe social que mais procurava por este recurso.

\section{RESULTADOS E DISCUSSÃO}

Foi constatado que nos quatro municípios onde foram aplicados os questionários a população utiliza remédios fitoterápicos. Os entrevistados fazem uso destes para a cura e combate das mais diversas enfermidades.

Nos municípios de Campo Grande e Umarizal 85\% dos clientes que procuram às farmácias para a compra dos remédios são mulheres e $15 \%$ apenas são homens. Já nos municípios de Caraúbas e Martins a freqüência é de 75\% mulheres e $25 \%$ homens que comparecem no ato da compra, isto se deve ao fato de que as mães de família são as responsáveis pela administração do lar e assim respondem pelas maiores porcentagens.

No que diz respeito ao consumo, no município de Campo Grande 60\% dos usuários são do sexo feminino e $40 \%$ do sexo masculino, estando numa faixa etária que vai dos 10 anos de idade à terceira idade, tendo maior representatividade dos 21-30 anos (50\%), seguido dos 31-45 anos (30\%), acima de 46 anos (15\%) e 10-20 anos (5\%). 


\section{REVISTA VERDE DE AGROECOLOGIA E DESENVOLVIMENTO SUSTENTÁVEL GRUPO VERDE DE AGRICULTURA ALTERNATIVA (GVAA)}

Nos municípios de Caraúbas, Martins e Umarizal $70 \%$ dos usuários são do sexo feminino e $30 \%$ masculino, os mesmos encontrando-se numa faixa etária que vai dos 10 anos à terceira idade. Mostram-se mais representativas pessoas com faixa etária dos 2130 anos (60\%), seguida dos 31-45 anos (15\%), 10-20 anos (10\%) e pessoas acima de 46 anos (5\%).

Relacionado ao uso repetitivo dos medicamentos, em todos os municípios $65 \%$ dos entrevistados já vêm utilizando os mesmos por mais de uma vez, e afirmam sempre que necessário recorrem aos mesmos deixando de lado os medicamentos convencionais. Os demais entrevistados (35\%), afirmam ser a primeira vez que recorrem ao uso destes medicamentos e que o uso é indicado por amigos, parentes ou vizinhos que já os utilizam e que têm obtido resultados satisfatórios quanto à cura das mais diversas enfermidades.

As classes sociais abrangidas são as mais diversas. Nos municípios de Caraúbas e Umarizal 70\% dos clientes pertencem às classes média e baixa e 30\% são da classe alta. Em Campo Grande, 50\% dos clientes são da classe média e baixa e $50 \%$ da classe alta. Em Martins, podemos constatar que $75 \%$ dos clientes pertencem à classe alta. Esse quadro deve-se ao fato que a renda mensal que essas famílias dispõem para sobreviver está em torno de um salário mínimo, assim, a grande maioria recorre a estes medicamentos por apresentarem preços mais acessíveis, sem, no entanto perderem a qualidade e apresentarem resultados iguais ou superiores àqueles oferecidos em farmácias convencionais, estando ainda atrelado a isto a confiabilidade que as pessoas depositam em medicamentos naturais, pois acreditam que ao fazerem uso destes estão se isentando direta ou indiretamente da ingestão de produtos químicos que se encontram embutidos nos medicamentos convencionais. Além do mais, paralelo aos fatores econômicos estão os culturais, o uso de plantas medicinais no combate as enfermidades se trata de um costume transmitido geração após geração, e ainda merece destaque a recomendação por parte médica.

São muitos os medicamentos disponíveis nas farmácias fitoterápicas e que se estendem as mais diversas enfermidades, na tabela 1 em anexo segue listagem dos medicamentos disponíveis, a planta utilizada em sua formulação e a doença à qual é recomendado o uso.

\section{CONCLUSÕES}

Em todos os municípios a população utiliza remédios fitoterápicos.

A maioria dos clientes e usuários são mulheres na faixa etária de 21-30 anos.
$65 \%$ dos entrevistados usaram estes medicamentos pela primeira vez e continuam usando-os.

Nos municípios de Campo Grande, Caraúbas e Umarizal as classes sociais beneficiadas são média e baixa, enquanto que em Martins a classe beneficiada é a alta com $75 \%$ dos clientes.

As pessoas buscam estes medicamentos pelo fato de serem mais acessíveis e apresentam resultados iguais ou superiores aos medicamentos convencionais.

\section{AGRADECIMENTOS}

A Associação de Produtos Naturais Longa Vida pela permissão e colaboração com este estudo.

\section{LITERATURA CITADA}

BRANDÃO, M. G. L.; FREIRE, N.; VIANNA-SOARES, C. D. Vigilância de fitoterápicos em Minas Gerais. Verificação da qualidade de diferentes amostras comerciais de camomila. Cadernos de Saúde Pública, v. 14, n. 3, p. 613616, 1998.

CARVALHO, V.M. de; et al. Uso e cultivo de plantas medicinais em residências de cidades do norte do Estado do Paraná. $45^{\circ}$ Congresso Brasileiro de Olericultura, $15^{\circ}$ Congresso Brasileiro de Plantas Ornamentais, $2^{\circ}$ Congresso Brasileiro de Cultura de Tecidos de Plantas, Fortaleza-CE, Brasil. Agosto/2005.

HARRI, L.R.; MATOS, F.J.A. Plantas Medicinais no Brasil: nativas e exóticas. Nova

Odessa - SP: Instituto plantarum, 2002. 544 p.

HOAREAU, L.; DASILVA, E. Medicinal plants: a reemerging health aid. Electronic Journal of Biotechnology, v.2, n.2, p. 57-70, ago. 1999. ISSN 0717-3458.

MARTINS, E.R. et al. Plantas Medicinais. Viçosa: UFV, Imprensa Universitária, 1995, 22p.

MATOS, F. J. A. Farmácias Vivas. Ed. Universidade Federal do Ceará, Fortaleza, 1998.

MING, L. C.; SILVA, S. M. P.; SILVA, M. A. S.; HIDALGO, A. F.; MARCHESE, J. A.; REIS, M.S. dos. Manejo sustentado de plantas medicinais em ecossistemas tropicais. In: DI STASI, L. C. (organizador). Plantas Medicinais: arte e ciência. Um guia de estudo 
multidisciplinar. 1a. ed. São Paulo, Ed. Unesp. 1996, p. 199-215.

RODRIGUES, V. E. G.; CARVALHO, D. A. Levantamento Etnobotânico de Plantas Medicinais do Domínio Cerrado na Região do Alto Rio Grande Minas Gerais. Ciências Agrotécnica, v. 25, n.1, 2001.

SIMÕES, C. M. O., MENTZ, L.A., SCHENKEL, E.P., IRGANG, B.E., STEHMANN, J.R. Plantas da Medicina Popular no Rio Grande do Sul. Porto Alegre, Ed. Da Universidade UFRGS, 1989, 174 p. 
REVISTA VERDE DE AGROECOLOGIA E DESENVOLVIMENTO SUSTENTÁVE

GRUPO VERDE DE AGRICULTURA ALTERNATIVA (GVAA)

ANEXOS

TABELA 1: Medicamentos disponíveis nas farmácias fitoterápicas e que são utilizados para o combate as doenças

\begin{tabular}{|c|c|c|}
\hline MEDICAMENTOS & PLANTA UTILIZADA & INDICAÇÃO \\
\hline Sabonete de Aroeira & $\begin{array}{l}\text { Aroeira (Astronium fraxcinifolium) } \\
\text { Confrei (Symphytum officinalis L.) } \\
\text { Alecrim (Rosmarinus officinalis L.) }\end{array}$ & $\begin{array}{l}\text { Limpeza de pele, manchas e } \\
\text { acne. }\end{array}$ \\
\hline Sabonete de Juazeiro & Juazeiro (Ziyiphus joazeiro L.) & Caspa e queda de cabelo. \\
\hline Xarope de Angico e Jatobá & $\begin{array}{l}\text { Angico (Anadenanthera macrocarpa } \\
\text { BENTH.) } \\
\text { Jatobá (Hymenaea courbalium L.) } \\
\text { Beterraba (Betta vulgaris) }\end{array}$ & Anemia, gripe e tosse. \\
\hline Mel de Cupim & $\begin{array}{l}\text { Jatobá (Hymenaea courbalium L.) } \\
\text { Angico (Anadenanthera macrocarpa } \\
\text { BENTH.) } \\
\text { Malvarisco (Malva silvestris L.) } \\
\text { Ipepaconha (Cechaelis ipecacuanha) } \\
\text { Cupim }\end{array}$ & Expectorante e anti-gripal. \\
\hline Elixir de Espinheira Santa & $\begin{array}{l}\text { Espinheira Santa (Maytenus ilicifolis Mart. } \\
\text { Ex Reiss.) } \\
\text { Jatobá (Hymenaea courbalium L.) } \\
\text { Unha de Gato (Uncarea tumetosa) } \\
\text { Arnica Vegetal (Arnica montana L.) } \\
\text { Erva Cidreira (Lippia alba (Mill) N.E.BR.) }\end{array}$ & Gastrite e úlcera. \\
\hline Elixir de Hortelã & $\begin{array}{l}\text { Hortelã (Mentha vilosa L.) } \\
\text { Agrião (Nasturpium officinale RICH) } \\
\text { Gengibre (Zingiber officinale Roscoe) }\end{array}$ & Faringite e amidalite \\
\hline Aroclim Xarope & $\begin{array}{l}\text { Aroeira (Astronium fraxcinifolium) } \\
\text { Alecrim (Rosmarinus officinalis L.) } \\
\text { Jatobá (Hymenaea courbalium L.) } \\
\text { Mel de Abelha }\end{array}$ & Inflamação de ovário \\
\hline Óleo de Angico & $\begin{array}{l}\text { Angico } \text { (Anadenanthera macrocarpa } \\
\text { BENTH). }\end{array}$ & $\begin{array}{lr}\text { Interno: bronquite } & \text { asmática, } \\
\text { tosse, jipe, intestino paralisado, } \\
\text { alergias em geral. Externo: } \\
\text { convulsões, } \\
\text { cicatrizante. }\end{array}$ \\
\hline Óleo de Coco & Coco (Cocos nucifera L.) & $\begin{array}{l}\text { Cicatrizante, anti-gripal } \\
\text { problemas de garganta. }\end{array}$ \\
\hline Óleo de Girassol & Girassol (Helianthus annus) & Uso ortopédico e cicatrizante. \\
\hline Óleo de Alecrim & Alecrim (Rosmarinus officinalis L.) & Dores reumáticas. \\
\hline Óleo de Gengibre & Gengibre (Zingiber officinale Roscoe) & Bronquite, rouquidão e asma. \\
\hline Óleo de Linhaça & Linhaça (Linum usitatissimum) & Hemorróidas e cólicas. \\
\hline
\end{tabular}


REVISTA VERDE DE AGROECOLOGIA E DESENVOLVIMENTO SUSTENTÁVE

GRUPO VERDE DE AGRICULTURA ALTERNATIVA (GVAA)

\begin{tabular}{|c|c|c|}
\hline Óleo de Aniroba & Andiroba (Carapa guianensie) & Dermatológico. \\
\hline Óleo de Carrapateira & Carrapateira (Ricinus sp.) & Laxante e convulsão de pele. \\
\hline Óleo de Gergelim & Gergelim (Sesamum indicum L.) & $\begin{array}{l}\text { Dores musculares, problemas de } \\
\text { coluna e anti-reumático. }\end{array}$ \\
\hline Óleo de Copaíba & Copaíba (Copaifera sp.) & $\begin{array}{l}\text { Dores musculares, cicatrizante e } \\
\text { úlceras. }\end{array}$ \\
\hline Óleo de Hortelã & Hortelã (Mentha vilosa L.) & $\begin{array}{l}\text { Dores musculares, dores de } \\
\text { cabeça, funcionamento de } \\
\text { intestino, analgésico, digestivo, } \\
\text { anestésico. }\end{array}$ \\
\hline Óleo de Eucalipto & Eucalipto (Eucaliptus globulus Labill.) & $\begin{array}{l}\text { Dores musculares, analgésico, } \\
\text { cicatrizante, dores de cabeça, } \\
\text { anestésico. }\end{array}$ \\
\hline Xarope para Verminoses & $\begin{array}{l}\text { Hortelã (Mentha vilosa L.) } \\
\text { Batata de Purga (Operculina macrocarpa L.) } \\
\text { Mastruz (Chenopodium ambrosioides L.) } \\
\text { Abóbora (Curcubita pepo L.) }\end{array}$ & Vermífugo. \\
\hline Comprimidos para Verminoses & $\begin{array}{l}\text { Hortelã (Mentha vilosa L.) } \\
\text { Batata de Purga (Operculina macrocarpa L.) } \\
\text { Mastruz (Chenopodium ambrosioides L.) } \\
\text { Abóbora (Curcubita pepo L.) }\end{array}$ & Vermífugo. \\
\hline Infusão para Sinusite & $\begin{array}{l}\text { Alecrim (Rosmarinus officinalis L.) } \\
\text { Cumarú (Dipteryx odorata (AUBL.) Willd) } \\
\text { Eucalipto (Eucaliptus globulus Labill.) } \\
\text { Canela (Cynnamomum zeylanicum Ness.) } \\
\text { Hortelã (Mentha spicalata L.) }\end{array}$ & Sinusite. \\
\hline Pomada de Romã & Romã (Punica granatum L.) & $\begin{array}{l}\text { Acne, furúnculos, tumores e } \\
\text { unheiras. }\end{array}$ \\
\hline Pomada para Rachaduras nos Pés & $\begin{array}{l}\text { Aroeira (Astronium fraxcinifolium) } \\
\text { Confrei (Symphytum officinalis L.) } \\
\text { Alecrim (Rosmarinus officinalis L.) }\end{array}$ & Rachaduras nos pés. \\
\hline Tintura de Aroeira & Aroeira (Astronium fraxcinifolium) & $\begin{array}{l}\text { Úlcera de pele, cicatrização de } \\
\text { ferimentos, reumatismos e } \\
\text { tumores. }\end{array}$ \\
\hline Tintura de Cajueiro & $\begin{array}{l}\text { Casca de Cajueiro (Anacardium occidentale } \\
\text { L.) }\end{array}$ & $\begin{array}{l}\text { Ferimentos, reumtismos, } \\
\text { verrugas, equizemas. }\end{array}$ \\
\hline Farinha de Linhaça & Sementes de Linhaça (Linum usitatissimum) & $\begin{array}{l}\text { Funcionamento de intestino, } \\
\text { controle de colesterol. }\end{array}$ \\
\hline Pomada para Hemorróidas & $\begin{array}{l}\text { Melão de São Caetano (Mormodica } \\
\text { charantuia L.) }\end{array}$ & Combate à hemorróidas. \\
\hline
\end{tabular}


REVISTA VERDE DE AGROECOLOGIA E DESENVOLVIMENTO SUSTENTÁVE

GRUPO VERDE DE AGRICULTURA ALTERNATIVA (GVAA)

Babosa (Aloe vera L.)

Pomada de Confrei

omada de Aroeira

abonete Anti-Micose

Solução Cicatrizante

Tintura de Pega-Pinto

Solução Anti-Micose
Confrei (Symphytum officinalis L.) Aroeira (Astronium fraxcinifolium) Alecrim (Rosmarinus officinalis L.)

Aroeira (Astronium fraxcinifolium)

Melão de
charantuia L.)

Babosa (Aloe vera L.)

Aroeira (Astronium fraxcinifolium)

Confrei (Symphytum officinalis L.)

Ameixa (Prumus domestica)

Pega-Pinto (Boerhaavia diffusa)

Melão de

charantuia L.)
Babosa (Aloe vera L.)
Descongestionamento nasal.

Cicatrizante,analgésico, controle de mancha.

Cicatrizante para uso externo.

Anti-próstata e cicatrizante.

Anti-Micose. 